\title{
Tunable Broadband Transparency of Macroscopic Quantum Superconducting Metamaterials
}

\author{
Daimeng Zhang, ${ }^{1,2, *}$ Melissa Trepanier, ${ }^{2}$ Oleg Mukhanov, ${ }^{3}$ and Steven M. Anlage ${ }^{1,2}$ \\ ${ }^{1}$ Department of Electrical and Computer Engineering, University of Maryland, \\ College Park, Maryland 20742-3285, USA \\ ${ }^{2}$ Center for Nanophysics and Advanced Materials, Department of Physics, University of Maryland, \\ College Park, Maryland 20742-4111, USA \\ ${ }^{3}$ Hypres, Inc., 175 Clearbrook Road, Elmsford, New York 10523, USA
}

(Received 16 June 2015; published 18 December 2015)

\begin{abstract}
Narrow-band invisibility in an otherwise opaque medium has been achieved by electromagnetically induced transparency (EIT) in atomic systems. The quantum EIT behavior can be classically mimicked by specially engineered metamaterials via carefully controlled interference with a "dark mode." However, the narrow transparency window limits the potential applications that require a tunable wideband transparent performance. Here, we present a macroscopic quantum superconducting metamaterial with manipulative self-induced broadband transparency due to a qualitatively novel nonlinear mechanism that is different from conventional EIT or its classical analogs. A near-complete disappearance of resonant absorption under a range of applied rf flux is observed experimentally and explained theoretically. The transparency comes from the intrinsic bistability of the meta-atoms and can be tuned on and off easily by altering rf and dc magnetic fields, temperature, and history. Hysteretic in situ $100 \%$ tunability of transparency paves the way for autocloaking metamaterials, intensity-dependent filters, and fast-tunable power limiters.
\end{abstract}

DOI: 10.1103/PhysRevX.5.041045

\section{INTRODUCTION}

Controllable transparency in an originally opaque medium has been an actively studied topic. Among those efforts, electromagnetically induced transparency (EIT) in three-level atomic systems is one of the most compelling ideas [1-3]. A classical analog of EIT atomic systems is realized in structured metamaterials that have a radiative mode coupled to a trapped mode [4-6]. The interaction between two modes induces a narrow transparency window in which light propagates with low absorption, also creating strong dispersion and a substantial slowing of light $[1-4,6]$. Superconducting metamaterials, especially, exhibit intrinsic nonlinearity, thus opening the door for tunable electromagnetic transparency [7-11]. Nevertheless, most techniques that induce transparency require careful manipulation of coupling and only allow light to propagate with perfect transmission in a narrow frequency bandwidth inside a broad resonance feature. The narrow-band invisibility prevents conventionally induced transparency from applications requiring broadband invisibility. In this work, we demonstrate a microwave metamaterial with

*dmchang@umd.edu

Published by the American Physical Society under the terms of the Creative Commons Attribution 3.0 License. Further distribution of this work must maintain attribution to the author(s) and the published article's title, journal citation, and DOI.
Subject Areas: Condensed Matter Physics, Metamaterials, Superconductivity self-induced broadband and tunable transparency that arises from an altogether new mechanism.

Our macroscopic quantum superconducting metamaterial is made of radio-frequency superconducting quantum interference device (rf SQUID) meta-atoms. An rf SQUID is a macroscopic quantum version of the split-ring resonator in that the gap capacitance is replaced with a Josephson junction. The rf SQUID is sensitive to the applied rf and dc magnetic flux, and the scale of this response is the flux quantum $\Phi_{0}=h / 2 e=2.07 \times 10^{-15} \mathrm{Tm}^{2}$, where $h$ is Planck's constant and $e$ is the elementary charge. The rf SQUID combines two macroscopic quantum phenomena: magnetic flux quantization and the Josephson effect [12], making it extremely nonlinear and tunable $[9,13,14]$. Incorporating rf SQUIDs into metamaterials has received increasing attention [15-22]. The large-range resonance tunability by dc magnetic flux at low drive amplitude [19-22] and switchable multistability at high drive amplitude [22] of rf SQUID metamaterials have been studied experimentally and theoretically. Lazarides and Tsironis predict dynamical bistability of rf SQUID metamaterials in the intermediate-rf-flux regime considered here [18]. However, the tunable broadband response of an $\mathrm{rf}$ SQUID metamaterial in the intermediate-rf-flux range has not been systematically examined by other experiment or theory.

Here, we demonstrate that in the intermediate-rf-flux range the rf SQUID metamaterial develops self-induced broadband and tunable transparency that arises from the 
intrinsic nonlinearity of rf SQUIDs. Both experiment and simulation show that the resonance of this metamaterial largely disappears when illuminated with electromagnetic waves of certain power ranges. We can adroitly control the metamaterial to be transparent or opaque depending on stimulus scanning directions. Note that the hysteretic transparency property results from the nonlinear dynamics of rf SQUIDs that are nonhysteretic in the static limit. The degree of transparency can be tuned by temperature, rf, and dc magnetic field. We also discuss analytical and numerical models that reveal the origin of the effect and how to systematically control the transparency regime. The nonlinear transparency behavior should also extend to the quantum regime of superconducting quantum metamaterials interacting with a small number of photons [7,23-26]. The observed tunable transparency of the rf SQUID metamaterial offers a range of previously unattainable functionalities because it acts effectively as a three-terminal device. New applications include wideband power limiters for direct-digitizing $\mathrm{rf}$ receivers [27], gain-modulated antennas [28], rf pulse shaping for qubit manipulation, tunable intensity-limiting filters, and the novel concept of a metamaterial that spontaneously reduces its scattering cross section to near zero (autocloaking), depending upon stimulus conditions.

\section{RESULTS}

\section{A. Broadband transparency at a fixed applied dc flux}

We measure the transmission and reflection of our metamaterial samples positioned in a rectangular waveguide so that the rf magnetic field of the propagating wave is perpendicular to the SQUID loop [Fig. 1(a)] [21] (see the Supplemental Material [29]). Measured transmission of a single meta-atom as a function of frequency and rf flux $\Phi_{\mathrm{rf}}$ at a temperature of $4.6 \mathrm{~K}$ under $0 \mathrm{dc}$ flux is shown in Fig. 1(b). Red features denote the resonance absorption dips of the meta-atom. At low input rf flux, the resonance is strong at $19 \mathrm{GHz}$ [21]. The horizontal splitting in the resonance is due to the standing waves in the waveguide created by impedance mismatches in the system. The strength of the low-rf-flux resonance can be enhanced by decreasing temperature (higher resistance in the Josephson junction and thus higher quality factor) and increasing the filling fraction of the metamaterial inside the waveguide by using a large array [21]. In the intermediaterf-flux range, the resonance shifts to lower frequency and systematically fades away $\left(\left|S_{21}\right|=0 \mathrm{~dB}\right)$ through the entire frequency range of single-mode propagation through the waveguide. At an upper critical rf flux, a strong resonance abruptly appears at the geometrical resonance frequency $\omega_{\text {geo }} / 2 \pi=13.52 \mathrm{GHz}$ for a single rf SQUID. We employ the nonlinear dynamics of an rf SQUID to numerically calculate transmission (see the Supplemental Material [29])
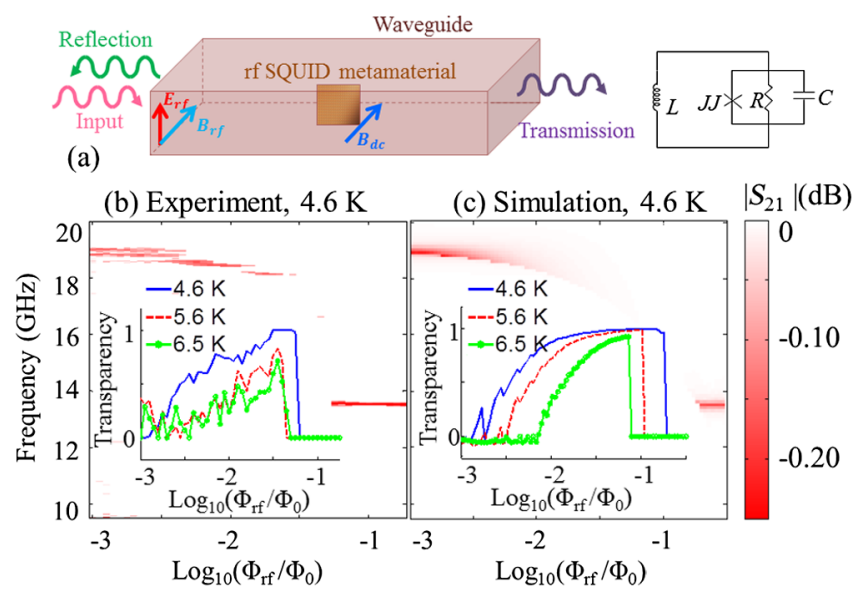

FIG. 1. (a) Schematic of the experimental setup for measuring the transmission of rf SQUID metamaterial oriented perpendicular to the rf magnetic field inside a rectangular waveguide. The dc magnetic field is applied by sending current into a superconducting coil outside the waveguide. The inset of (a) is the resistively and capacitively shunted junction model of an $\mathrm{rf}$ SQUID. Transmission of a single meta-atom at $4.6 \mathrm{~K}$ depending on frequency and rf flux is shown in (b) experiment and (c) simulation. The red (dark) feature denotes the resonance dip. Insets plot the transparency as a function of input power under 4.6 K (solid blue lines), 5.6 K (dashed red lines), and 6.5 K (dotted green lines) both for (b) experiment and (c) simulation.

shown in Fig. 1(c), which shows the same transparency behavior.

We define a normalized transparency level that quantitatively determines the degree of resonance absorption compared to the low-rf-flux absorption (see the Supplemental Material [29]). High transparency indicates a weak resonance absorption. The extracted transparency shows a clear onset rf flux for transparency and an upper critical rf flux determining the abrupt end of transparency [insets of Figs. 1(b) and 1(c)]. The transparency approaches 1.0 between these rf flux values. The measurements are taken at 4.6, 5.6, and $6.5 \mathrm{~K}$; at lower temperature, both experiment and simulation show a larger range of transparency as well as a higher degree of transparency.

\section{B. Hysteretic transparency}

Collecting rf SQUID meta-atoms into a metamaterial preserves the self-induced broadband transparency performance. Figure 2 illustrates the transmission of an $11 \times 11 \mathrm{rf}$ SQUID array metamaterial (see the Supplemental Material for parameters [29]) with interactions among the metaatoms. The metamaterial is stimulated at fixed frequency while the rf flux amplitude is scanned under nominally 0 applied dc flux at $4.6 \mathrm{~K}$. Again, there are some horizontal features resulting from metamaterial interacting with the standing waves in the waveguide. The resonance is almost invisible as the input rf flux increases continuously through the transparency range [Fig. 2(a)]. 


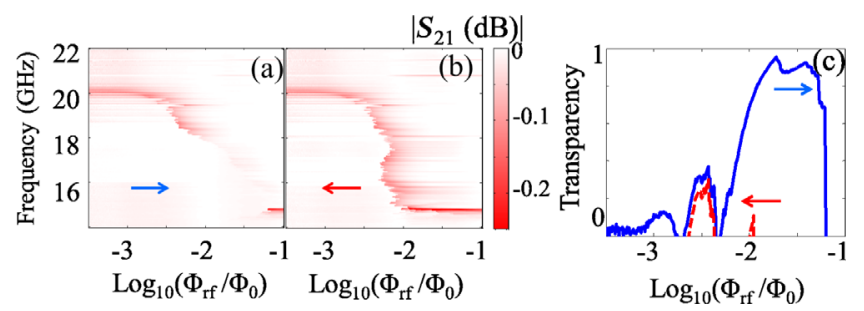

FIG. 2. Measured transmission as a function of frequency and applied rf flux for an $11 \times 11$ array rf SQUID metamaterial, when the input rf flux (a) keeps increasing and (b) continuously decreases. (c) Extracted transparency values for sweep directions (a) and (b). The arrows denote the rf flux scanning directions. The temperature is $4.6 \mathrm{~K}$.

However, a reverse rf flux scan renders an opaque behavior [Fig. 2(b)]: The resonance is strong across all rf flux values. Quantitatively, the transparency value reaches 0.9 in the forward sweep and is below 0.3 for the backward sweep [Fig. 2(c)]. We did numerical simulations on this metamaterial, and they show the same hysteretic transparent or opaque behavior. Similar hysteresis is also observed for measurements and simulations of a single rf SQUID meta-atom. These observations mean that transparency can be turned on and off depending on the stimulus scan direction and metamaterial history.

\section{Transparency and bistability}

The origin of the nonlinear transparency is the intrinsic bistability of the rf SQUID. The gauge-invariant phase difference of the macroscopic quantum wave function across the Josephson junction $\delta(t)$ and its time dependence determine essentially all properties of the rf SQUID and the associated metamaterial (see the Supplemental Material [29]). In simulation, we find that $\delta(t)$ is almost purely sinusoidal; the amplitudes of the higher harmonics are less than $1 \%$ of the fundamental resonance amplitude for all drive amplitudes considered here. The amplitude of the gauge-invariant phase oscillation on resonance as a function of rf flux for a forward stimulus sweep $\left(\delta_{\mathrm{LH}}\right)$ is lower than the amplitude for a reverse sweep $\left(\delta_{\mathrm{HL}}\right)$ above the onset of bistability [Fig. 3(a)]. The lower gauge-invariant phase amplitude results in a smaller magnetic susceptibility and thus a reduction of resonant absorption. The relation between the resonance strength (degree of transparency) and $\delta_{\mathrm{LH}}$ is shown in Fig. 3(b) for the transparent state. The onset rf flux of transparency coincides with the abrupt reduction of $\delta_{\mathrm{LH}}-\Phi_{\mathrm{rf}}$ slope and the onset of bistability.

We can apply the Duffing oscillator approximation to analytically predict the onset of bistability. For intermediate drive amplitude, an rf SQUID can be treated as a Duffing oscillator (Kerr oscillator) [23], which is a model widely adopted for studying Josephson parametric amplifiers [30,31]. This approximation suggests that when the drive amplitude reaches a critical value, the amplitude of the $\delta(t)$
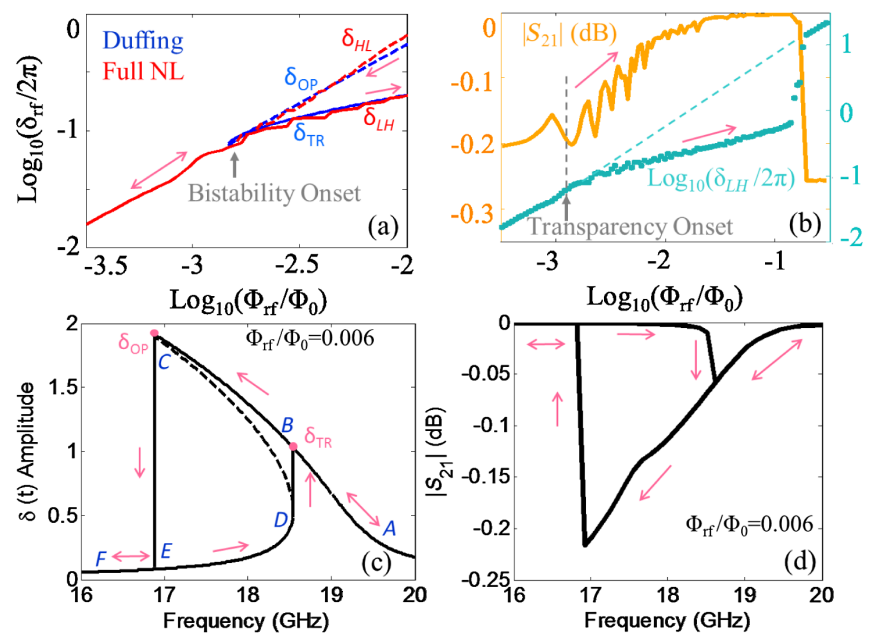

FIG. 3. Simulation results for a single rf SQUID in the bistable regime. The pink arrows denote the signal (frequency or rf flux amplitude) scanning directions. (a) The amplitude of gaugeinvariant phase $\delta(t)$ oscillation on resonance as a function of driving rf flux amplitude. Red curves denote the full nonlinear numerical results. The $\delta_{\mathrm{LH}}$ is the amplitude of $\delta(t)$ when the frequency or rf flux scans from low value to high value, and $\delta_{\mathrm{HL}}$ is the amplitude for a reverse scan. Blue curves are calculated analytically with the Duffing oscillator approximation. The $\delta_{\mathrm{OP}}$ and $\delta_{\mathrm{TR}}$ denote the analytic amplitudes for the opaque state and the transparent state, respectively. The gray arrow points out the onset of bistability. (b) The simulated transmission (orange curve, left $y$ axis) and the amplitude of $\delta(t)$ (green curve, right $y$ axis) on resonance as a function of rf flux for the transparent case during an upward-rf-flux scan. The dashed green line shows the $\delta(t)$ amplitude interpolated between the low-power and high-power limits. The gray arrow shows the onset rf flux for transparency. It is the same as the bistability onset. (c) The foldover resonance of the amplitude of $\delta(t)$ calculated in the Duffing oscillator approximation results in a bistable oscillation on resonance with amplitudes $\delta_{\mathrm{TR}}$ and $\delta_{\mathrm{OP}}$, respectively, at an rf flux of $0.006 \Phi_{0}$. The dashed curve plots the unstable solutions in the foldover resonance. (d) The numerically calculated transmission as a function of rf driving frequency at an rf flux of $0.006 \Phi_{0}$ shows that the frequency range of bistability is the same as analytically predicted in Duffing oscillator approximation in (c).

oscillation as a function of frequency is a foldover resonance [Fig. 3(c)], creating bistable oscillating states (see the Supplemental Material [29]). The amplitudes of the gauge-invariant phase-difference oscillation for the transparent state $\left(\delta_{\mathrm{TR}}\right)$ and the opaque state $\left(\delta_{\mathrm{OP}}\right)$ are calculated analytically for each rf flux and compared to the amplitudes $\delta_{\mathrm{LH}}$ and $\delta_{\mathrm{HL}}$ calculated numerically [Fig. 3(a)]. Both the onset of bistability and the amplitudes of the two states match very well. The bistability of $\delta(t)$ amplitudes explains the bistability of transmission observed in experiment and simulation [Fig. 3(d)]. The very good agreement between the Duffing oscillator and the full nonlinear numerical simulation allows us to study analytically how to enhance the transparency values and the transparency range. 
The onset rf flux value for transparency depends on several parameters. Higher resistance in the junction, higher capacitance, and higher critical current all give a lower onset rf flux for transparency (see the Supplemental Material [29]). Operating the metamaterial at a lower temperature increases the resistance and the critical current, thus decreasing the onset, explaining the modulation of onset by temperature observed in experiment and simulation (insets of Fig. 1). The applied dc flux has a more modest effect on the onset rf flux. With a dc flux of a quarter flux quantum, the single rf SQUID has an onset that is $13 \%$ smaller than the 0 -flux case (see the Supplemental Material [29]).

\section{Modulation of transparency by dc flux}

The dc flux has a strong modulation of the transparency upper critical rf flux. Above the upper critical rf flux, the rf SQUID experiences phase slips on each rf cycle and shows strong resonant absorption at the geometrical frequency $\omega_{\text {geo }} / 2 \pi$ [22]. We can determine the transparency upper critical edge when the driving frequency is fixed at the geometrical frequency $\omega_{\text {geo }} / 2 \pi$, while rf flux amplitude scans from $0.001 \Phi_{0}$ to $0.1 \Phi_{0}$. The sudden decrease of transmission denotes the upper critical rf flux in differing amounts of dc flux [Fig. 4(a)]. The numerical simulation is depicted in Fig. 4(b). There is a tunability of over a factor of 3.5 in transparency upper critical flux by varying dc flux through the sample. Note that the entire dc magnetic field variation in Fig. 4 is only $10 \mathrm{nT}$. The result shows that at a fixed frequency $\omega_{\text {geo }} / 2 \pi$, the meta-atom can be transparent or opaque depending very sensitively on the rf flux and dc flux.

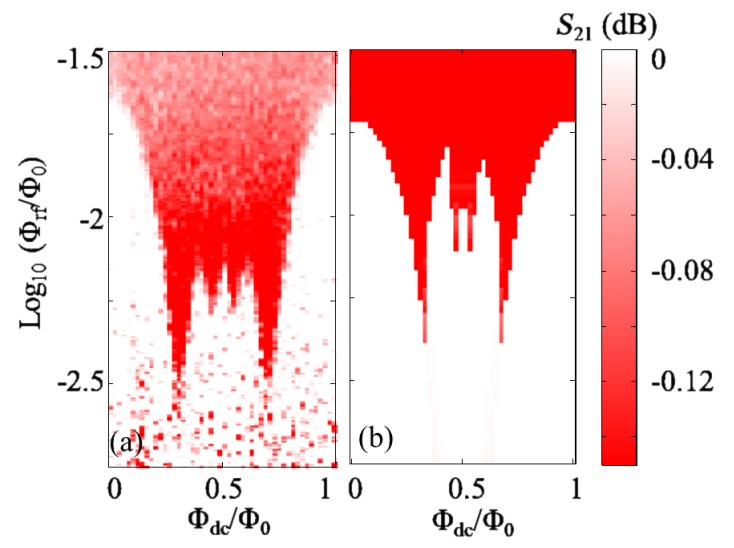

FIG. 4. The transmission as a function of rf flux and dc flux at the geometrical frequency $\omega_{\text {geo }} / 2 \pi$ of (a) experiment and (b) simulation for a single rf SQUID at $4.6 \mathrm{~K}$. The strong resonance absorption (red region) at the geometrical frequency determines the upper critical rf flux of transparency. The edge between the red region and the white region denotes the tuning of upper critical rf flux by applied dc flux. The experiment is taken with pulsed rf measurement with a duty cycle of $1 \%$ (see the Supplemental Material [29]). The pulse width is $2 \mu \mathrm{s}$, which is long enough to ensure that the rf SQUID achieves a steady state.

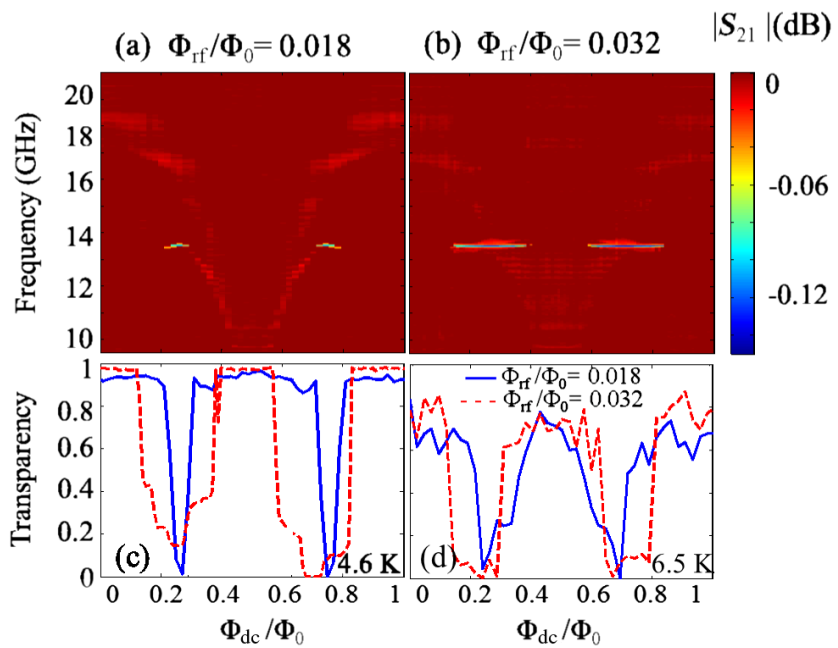

FIG. 5. The measured transmission at $4.6 \mathrm{~K}$ of a single $\mathrm{rf}$ SQUID meta-atom as a function of dc flux and frequency at different drive amplitudes: (a) rf flux $=0.018 \Phi_{0}$, (b) rf flux $=$ $0.032 \Phi_{0}$. Dark red is the perfect transmission background; light red and blue features denote the weak and strong resonance absorption, respectively. The extracted transparency values for $\mathrm{rf}$ flux amplitude of $0.018 \Phi_{0}$ (dashed red line) and $0.032 \Phi_{0}$ (solid blue line) are shown for (c) $4.6 \mathrm{~K}$ and (d) $6.5 \mathrm{~K}$. They both show a clear switchable transparent behavior by dc flux.

Also, at a fixed rf flux near the upper critical edge, the sample can be resonantly absorbing at $\omega_{\text {geo }}$ or transparent in the broadband frequency window depending sensitively on the applied dc flux. Figure 5 plots the experimental transmission as a function of dc flux and frequency when our sample is illuminated with an rf flux amplitude of $0.018 \Phi_{0}$ and $0.032 \Phi_{0}$, respectively. Strong absorption at the geometrical resonance appears around $\Phi_{0} / 4$ and $3 \Phi_{0} / 4 \mathrm{dc}$ flux values, while being broadband transparent near $0 \Phi_{0}$ and $0.5 \Phi_{0}$. At $\Phi_{0} / 4$ and $3 \Phi_{0} / 4 \mathrm{dc}$ flux values, the Josephson inductance diverges and the system naturally resonates at the geometrical frequency [21]. Figure 5(c) shows that higher-rf-flux values push the rf SQUID to be opaque under a larger range of dc flux, but the maximum value of transparency is higher than the lower-rf-flux case [Fig. 5(c)]. As the temperature increases, the transparency is weaker, as seen in Fig. 5(d). The tuning of transparency by dc flux at a fixed rf flux indicates again a switchable onand-off transparency behavior with small variations of dc flux for our meta-atom.

\section{DISCUSSION}

Up to this point, we have mainly discussed the results for a single rf SQUID because the transparency behavior of the rf SQUID metamaterial arises from the bistability of single meta-atoms. The rf SQUIDs in a metamaterial array show the same tunable broadband transparency properties as the single SQUID. The $11 \times 11$ array metamaterial not only 
preserves the rf flux-modulated transparency (Fig. 2) but also maintains the unusual dc flux-switchable transparency (see the Supplemental Material, Fig. S4 [29]). Disorder in the rf or dc flux slightly affects the degree of transparency in an rf SQUID metamaterial. For example, in experiments on an $11 \times 11$ array metamaterial, an intentionally introduced gradient of $0.11 \Phi_{0}$ applied dc flux across the array changes the peak transparency value from 0.94 (for the uniform applied flux case) to 0.91 . The interaction between rf SQUIDs in a 2D array has a more complicated effect on the resonance behavior. The interaction is always inductively negative, giving rise to a smaller effective geometrical inductance. As a result, the low-rf-flux resonant frequency at $0 \mathrm{dc}$ flux shifts to a higher frequency compared to the individual rf SQUID case. Also, the geometrical resonance frequency rises by a frequency shift larger than the increase of low-rf-flux resonant frequency. Thus, the interaction depresses the tunability range in resonant frequency via if flux. However, the tunability reduction is very small due to the limited packing density of the 2D rf SQUID arrays, and the metamaterials preserve the broadband tunable performance even with interaction (see the Supplemental Material [29]). Moreover, both numerical simulation and analytic approximation of coupled rf SQUID metamaterial suggest that the onset rf flux for transparency is nearly the same as the noncoupled case, while the end of the rf flux for transparency is decreased. Hence, the coupling between meta-atoms decreases the transparency rf flux range [32].

Note that at high drive amplitudes, the rf SQUID becomes multistable around the geometrical frequency $\omega_{\text {geo }}$ and resonates strongly at the geometrical resonance [33]. The tuning of the resonant frequency is thus absent in the multistable regime. The multistability has been studied in detail experimentally and theoretically [22]. Our work focuses instead on the modulation of resonance strength and resonance frequency as rf flux and dc flux vary for intermediate-rf-flux amplitudes, where the tunable transparency behavior is exclusively bistable and can be well explained by the Duffing oscillator approximation. The Duffing oscillator model offers an analytical prediction for the onset rf flux of bistability (see the Supplemental Material [29]) and an accurate approximation for the oscillation amplitudes. This allows us to study systematically the parameter dependence of the transparency tuning (see the Supplemental Material [29]).

The observed tunable transparency of the rf SQUID metamaterial offers a range of new previously unattainable functionalities, enabling new applications. The root cause of the tunable transparency comes from the intrinsic bistability of rf SQUIDs, which can be controlled in a number of ways. Some of these applications capitalize on the effective three-terminal device configuration in which transmission is modulated via a parameter, namely, the rf or the dc flux channel.
For example, one can design an rf transmitter where the input rf bias signal modulates the main channel rf transmission. Such a transmitter can be used in quantum computing to shape rf pulses for qubit control [34], particularly through the scanning direction hysteresis.

The self-induced transparency modulation feature can also lead to the presently unattainable gain-control capability in SQUID arrays, which are the basis for highly sensitive quantum antennas [28]. This would require engineering a linear transparency and rf power transfer function in the rf SQUID metamaterial.

Another application is in wideband direct-digitizing rf receivers, in which the rf SQUID metamaterial acts as an input power limiter to protect sensitive superconducting wideband digital $\mathrm{rf}$ receivers from strong jammers [27]. With frequency selectivity, one can devise tunable intensity-limiting filters to eliminate strong interference while remaining transparent for other frequencies.

The remarkable sharpness of transparency modulation by dc flux at a fixed applied rf flux amplitude (Fig. 5) gives an opportunity to use single-flux quantum (SFQ) logic to achieve fast transparency switching of the rf SQUID metamaterial $[21,35]$. This will enable a range of applications starting from SFQ-modulated digital communication transmitters to energy-efficient wireless data links between low-power cryogenic SFQ electronics and room-temperature semiconductor modules. Both of these applications are difficult to solve by means of conventional lowdissipation superconducting electronics.

\section{CONCLUSION}

In summary, we show that self-induced broadband transparency is observed in single rf SQUID meta-atoms and in rf SQUID metamaterials. The transparency arises from the bistability of individual SQUIDs. The transparency is hysteretic and switchable and can be tuned by temperature, dc, and rf magnetic flux, endowing the metamaterial with an "electromagnetic memory." The transparency range and level can be enhanced through numerous parameters under experimental control.

\section{ACKNOWLEDGMENTS}

This work is supported by the NSF-GOALI and OISE programs through Grant No. ECCS-1158644 and the Center for Nanophysics and Advanced Materials (CNAM). Funding for Open Access provided by the UMD Libraries Open Access Publishing Fund. We thank Masoud Radparvar, Georgy Prokopenko, Jen-Hao Yeh, and Tamin Tai for experimental guidance and helpful suggestions and Alexey Ustinov, Philipp Jung, Susanne Butz, Edward Ott, and Thomas Antonsen for helpful discussions. We also thank H. J. Paik and M. V. Moody for use of the pulsed tube refrigerator, as well as Cody Ballard and Rangga Budoyo for use of the dilution refrigerator. 
[1] S. E. Harris, J. E. Field, and A. Imamoğlu, Nonlinear Optical Processes Using Electromagnetically Induced Transparency, Phys. Rev. Lett. 64, 1107 (1990).

[2] S. H. Harris, Electromagnetically Induced Transparency, Phys. Today 50, No. 7, 36 (1997).

[3] M. Fleischhauer, A. Imamoglu, and J. P. Marangos, Electromagnetically Induced Transparency: Optics in Coherent Media, Rev. Mod. Phys. 77, 633 (2005).

[4] V. A. Fedotov, M. Rose, S. L. Prosvirnin, N. Papasimakis, and N. I. Zheludev, Sharp Trapped-Mode Resonances in Planar Metamaterials with a Broken Structural Symmetry, Phys. Rev. Lett. 99, 147401 (2007).

[5] S. D. Jenkins and J. Ruostekoski, Metamaterial Transparency Induced by Cooperative Electromagnetic Interactions, Phys. Rev. Lett. 111, 147401 (2013).

[6] C. Kurter, P. Tassin, L. Zhang, T. Koschny, A. P. Zhuravel, A. V. Ustinov, S. M. Anlage, and C. M. Soukoulis, Classical Analogue of Electromagnetically Induced Transparency with a Metal-Superconductor Hybrid Metamaterial, Phys. Rev. Lett. 107, 043901 (2011).

[7] A. A. Abdumalikov, O. Astafiev, A. M. Zagoskin, Yu. A. Pashkin, Y. Nakamura, and J. S. Tsai, Electromagnetically Induced Transparency on a Single Artificial Atom, Phys. Rev. Lett. 104, 193601 (2010).

[8] V. A. Fedotov, A. Tsiatmas, J. H. Shi, R. Buckingham, P. de Groot, Y. Chen, S. Wang, and N. I. Zheludev, Temperature Control of Fano Resonances and Transmission in Superconducting Metamaterials, Opt. Express 18, 9015 (2010).

[9] P. Jung, A. V. Ustinov, and S. M. Anlage, Progress in Superconducting Metamaterials, Supercond. Sci. Technol. 27, 073001 (2014).

[10] C. Kurter, P. Tassin, A. P. Zhuravel, L. Zhang, T. Koschny, A. V. Ustinov, C. M. Soukoulis, and S. M. Anlage, Switching Nonlinearity in a Superconductor-Enhanced Metamaterial, Appl. Phys. Lett. 100, 121906 (2012).

[11] G. P. Tsironis, N. Lazarides, and I. Margaris, Wide-Band Tuneability, Nonlinear Transmission, and Dynamic Multistability in SQUID Metamaterials, Appl. Phys. A 117, 579 (2014).

[12] M. Tinkham, Introduction to Superconductivity, 2nd ed. (McGraw-Hill, New York, 1996).

[13] S. M. Anlage, The Physics and Applications of Superconducting Metamaterials, J. Opt. 13, 024001 (2011).

[14] M. Lapine, I. V. Shadrivov, and Y. S. Kivshar, Colloquium: Nonlinear Metamaterials, Rev. Mod. Phys. 86, 1093 (2014).

[15] C. G. Du, H. Y. Chen, and S. Q. Li, Quantum Left-Handed Metamaterial from Superconducting Quantum-Interference Devices, Phys. Rev. B 74, 113105 (2006).

[16] N. Lazarides and G. P. Tsironis, rf Superconducting Quantum Interference Device Metamaterials, Appl. Phys. Lett. 90, 163501 (2007).

[17] A. I. Maimistov and I. R. Gabitov, Nonlinear Response of a Thin Metamaterial Film Containing Josephson Junctions, Opt. Commun. 283, 1633 (2010).

[18] N. Lazarides and G.P. Tsironis, Multistability and Self-Organization in Disordered SQUID Metamaterials, Supercond. Sci. Technol. 26, 084006 (2013).
[19] P. Jung, S. Butz, S. V. Shitov, and A. V. Ustinov, Low-Loss Tunable Metamaterials Using Superconducting Circuits with Josephson Junctions, Appl. Phys. Lett. 102, 062601 (2013).

[20] S. Butz, P. Jung, L. V. Filippenko, V. P. Koshelets, and A. V. Ustinov, A One-Dimensional Tunable Magnetic Metamaterial, Opt. Express 21, 22540 (2013).

[21] M. Trepanier, D. Zhang, O. Mukhanov, and S. M. Anlage, Realization and Modeling of Metamaterials Made of $r f$ Superconducting Quantum-Interference Devices, Phys. Rev. X 3, 041029 (2013).

[22] P. Jung, S. Butz, M. Marthaler, M. V. Fistul, J. Leppäkangas, V.P. Koshelets, and A. V. Ustinov, Multistability and Switching in a Superconducting Metamaterial, Nat. Commun. 5, 3730 (2014).

[23] L. S. Bishop, E. Ginossar, and S. M. Girvin, Response of the Strongly Driven Jaynes-Cummings Oscillator, Phys. Rev. Lett. 105, 100505 (2010).

[24] M. Boissonneault, J. M. Gambetta, and A. Blais, Improved Superconducting Qubit Readout by Qubit-Induced Nonlinearities, Phys. Rev. Lett. 105, 100504 (2010).

[25] M. D. Reed, L. DiCarlo, B. R. Johnson, L. Sun, D. I. Schuster, L. Frunzio, and R. J. Schoelkopf, High-Fidelity Readout in Circuit Quantum Electrodynamics Using the Jaynes-Cummings Nonlinearity, Phys. Rev. Lett. 105, 173601 (2010).

[26] P. Macha, G. Oelsner, J.-M. Reiner, M. Marthaler, S. André, G. Schön, U. Hübner, H.-G. Meyer, E. Il' ichev, and A. V. Ustinov, Implementation of a Quantum Metamaterial Using Superconducting Qubits, Nat. Commun. 5, 5146 (2014).

[27] O. A. Mukhanov, D. Kirichenko, I. V. Vernik, T. V. Filippov, A. Kirichenko, R. Webber, V. Dotsenko, A. Talalaevskii, J. C. Tang, A. Sahu, P. Shevchenko, R. Miller, S. B. Kaplan, S. Sarwana, and D. Gupta, Superconductor Digital-rf Receiver Systems, IEICE Trans. Electron. E91-C, 306 (2008).

[28] O. Mukhanov, G. Prokopenko, and R. Romanofsky, Quantum Sensitivity: Superconducting Quantum Interference Filter-Based Microwave Receivers, IEEE Microw. Mag. 15, 57 (2014).

[29] See Supplemental Material at http://link.aps.org/ supplemental/10.1103/PhysRevX.5.041045 for details of the samples and experiment, theoretical calculations, and more experimental results.

[30] R. Vijay, M. H. Devoret, and I. Siddiqi, Invited Review Article: The Josephson Bifurcation Amplifier, Rev. Sci. Instrum. 80, 111101 (2009).

[31] O. Yaakobi, L. Friedland, C. Macklin, and I. Siddiqi, Parametric Amplification in Josephson Junction Embedded Transmission Lines, Phys. Rev. B 87, 144301 (2013).

[32] M. Trepanier, D. Zhang, O. Mukhanov, and S. M. Anlage, Meta-Atom Interactions and Coherent Response in Superconducting Macroscopic Quantum Metamaterials (to be published).

[33] P. Jung, Ph.D. thesis, Karlsruher Institut für Technologie (KIT), 2014, http://www.ksp.kit.edu/download/1000043835.

[34] A. W. Cross and J. M. Gambetta, Optimized Pulse Shapes for a Resonator-Induced Phase Gate, Phys. Rev. A 91, 032325 (2015).

[35] O. A. Mukhanov, Energy-Efficient Single Flux Quantum Technology, IEEE Trans. Appl. Supercond. 21, 760 (2011). 